\title{
Equilibration kinetics in isolated and membrane-bound photosynthetic reaction centers upon illumination: a method to determine the photoexcitation rate
}

\author{
Anthony J. Manzo • Alexander O. Goushcha • \\ Yuri M. Barabash • Valery N. Kharkyanen • \\ Gary W. Scott
}

Received: 13 February 2009/ Accepted: 9 June 2009/Published online: 4 July 2009

(c) The Author(s) 2009. This article is published with open access at Springerlink.com

\begin{abstract}
Kinetics of electron transfer, following variation of actinic light intensity, for photosynthetic reaction centers (RCs) of purple bacteria (isolated and membrane-bound) were analyzed by measuring absorbance changes in the primary photoelectron donor absorption band at $865 \mathrm{~nm}$. The bleaching of the primary photoelectron donor absorption band in RCs, following a sudden increase of illumination from the dark to an actinic light intensity of $I_{\text {exp }}$, obeys a simple exponential law with the rate constant $\left(\alpha I_{\exp }+k_{\mathrm{rec}}\right)$, in which $\alpha$ is a parameter relating the light intensity, measured in $\mathrm{mW} / \mathrm{cm}^{2}$, to a corresponding theoretical rate in units of reciprocal seconds, and $k_{\text {rec }}$ is the effective rate constant of the charge recombination in the photosynthetic RCs. In this work, a method for determining the $\alpha$ parameter value is developed and experimentally verified for isolated and membrane-bound RCs, allowing for rigorous modeling of RC macromolecule dynamics under varied photoexcitation conditions. Such modeling is necessary for RCs due to alterations of the forward photoexcitation rates and relaxation rates caused by illumination history and intramolecular structural dynamics effects. It is demonstrated that the classical Bouguer-Lambert-Beer formalism can be applied for the samples with relatively low scattering, which is not
\end{abstract}

A. J. Manzo · A. O. Goushcha · G. W. Scott ( $\square)$

Department of Chemistry, UC Riverside, Riverside, CA 92521,

USA

e-mail: gary.scott@ucr.edu

A. O. Goushcha · Y. M. Barabash · V. N. Kharkyanen Institute of Physics, Nat. Acad. Sci. Ukraine, Kyiv, Ukraine

A. J. Manzo

Department of Chemistry, University of Michigan, Ann Arbor, MI 48109, USA necessarily the case with strongly scattering media or high light intensity excitation.

Keywords Rhodobacter sphaeroides - Reaction center . Electron transfer . Excitation rate

\begin{tabular}{|c|c|}
\hline \multicolumn{2}{|c|}{ Abbreviations } \\
\hline $\mathrm{RC}$ & Reaction center \\
\hline$I_{\exp }$ & Experimental excitation intensity \\
\hline$\alpha$ & $\begin{array}{l}\text { Alpha parameter and/or light intensity scaling } \\
\text { parameter }\end{array}$ \\
\hline$k_{\mathrm{rec}}$ & Recombination rate constant \\
\hline BLB & Bouguer-Lambert-Beer \\
\hline ET & Electron transfer \\
\hline$P$ & $\begin{array}{l}\text { Bacteriochlorophyll dimmer (also primary } \\
\text { dimmer and/or donor) }\end{array}$ \\
\hline$B P h$ & Bacteriopheophytin \\
\hline$P^{+}$ & Oxidized bacteriochlorophyll dimmer \\
\hline$Q_{A}$ & Primary quinone \\
\hline$Q_{B}$ & Secondary quinone \\
\hline$Q_{A}^{-}$ & Reduced primary quinone \\
\hline$Q_{B}^{-}$ & Reduced secondary quinone \\
\hline$\tau_{A}$ & $\begin{array}{l}\text { Lifetime of fast charge recombination } \\
\text { component of RC }\end{array}$ \\
\hline$\tau_{B}$ & $\begin{array}{l}\text { Lifetime of slow charge recombination } \\
\text { component of } \mathrm{RC}\end{array}$ \\
\hline$C_{A}$ & $\begin{array}{l}\text { Amplitude of fast charge recombination } \\
\text { component of } \mathrm{RC}\end{array}$ \\
\hline$C_{B}$ & $\begin{array}{l}\text { Amplitude of slow charge recombination } \\
\text { component of } \mathrm{RC}\end{array}$ \\
\hline$C_{O}$ & Constant (offset) \\
\hline $\mathrm{CW}$ & Continuous wave photoexcitation \\
\hline$I$ & $\begin{array}{l}\text { Theoretical light intensity (frequency of } \\
\text { photoexcitation of RC per unit time) }\end{array}$ \\
\hline
\end{tabular}


RC01 Membrane-bound antennae-free Rhodobacter (Rb.) sphaeroides strain R26

LDAO Lauryl- $N, N$,-dimethylamine- $N$-oxide

$A_{280} \quad$ Absorbance at $\lambda=280 \mathrm{~nm}$

$A_{800} \quad$ Absorbance at $\lambda=800 \mathrm{~nm}$

Tris-HCl Tris-hydroxy-methyl-amine-methane

EDTA Ethylenediaminetetraacetic acid

Q-4 Ubiquinone isoprene homologue

Q-10 Ubiquinone-10

LH1 Light harvesting antenna complex 1

LH2 Light harvesting antenna complex 2

$\mathrm{bc}_{1} \quad$ Bacteriochlorophyll $\mathrm{bc}_{1}$ complex

$\lambda \quad$ Wavelength

$\lambda_{\mathrm{cw}} \quad$ Continuous wave excitation wavelength

ca. Approximately

$D A \quad$ Charge neutral donor-acceptor state

$D^{+} A^{-} \quad$ Charge-separated donor-acceptor state

$k_{A P} \quad$ Rate constant for charge recombination from primary quinine to primary donor

$k_{A} \quad$ The charge recombination rate from primary quinone to dimmer, equal $k_{A P}$

$k_{A B} \quad$ Rate constant for charge transfer from primary quinone to secondary quinone

$k_{B A} \quad$ Rate constant for charge transfer from secondary quinone to primary quinone

$k_{B} \quad \approx k_{A P} k_{k_{A B}}$ the charge recombination rate from secondary quinone to dimmer

$\rho(t, D) \quad$ Normalized time dependant population of charge neutral state

$\rho(t, A) \quad$ Normalized time dependant population of charge separated state

$\kappa \quad I+k_{\text {rec }}$, electronic equilibration rate constant

$t \quad$ Time

$k_{\text {rec }}^{\prime} \quad$ Effective charge recombination rate constant

$\tau_{d} \quad\left(k_{\text {rec }}^{\prime}\right)^{-1}$

$\tau_{e l} \quad\left(I+k_{\text {rec }}^{\prime}\right)^{-1}$

$\tau_{s} \quad$ Single exponential decay lifetime

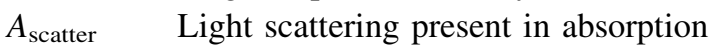
measurement

$C_{s} \quad$ Light scattering amplitude

$K_{s} \quad$ Light scattering parameter

\section{Introduction}

Upon illumination of a photosynthetic reaction center (RC) the bacteriochlorophyll dimer $P$ is excited and charge separation occurs followed by electron transfer along the active branch of electron acceptors in the direction of the secondary quinone acceptor $Q_{B}$ (see, e.g., Hoff and Deisenhofer (1997) for a review). Electron transfer (ET) initially occurs from the excited dimer to a bacteriopheophytin $B P h$ with an efficiency of $\sim 1$, in $\sim 2-4$ ps. Subsequent ET from the $B P h$ to the primary quinone acceptor $Q_{A}$ occurs in $\sim 150$ $2,000 \mathrm{ps}$. This is followed by ET to the secondary quinone acceptor $Q_{B}$, in a transfer time of $\sim 10^{-4} \mathrm{~s}$ (Kleinfeld et al. 1984a). For RCs that lack a quinone at the secondary acceptor site, charge recombination from $Q_{A}^{-}$to the photo oxidized $P^{+}, P^{+} Q_{A}^{-} \rightarrow P Q_{A}$, occurs with a rate constant of $\sim 10 \mathrm{~s}^{-1}$, increasing by 3-5 times under steady-state illumination conditions (Kleinfeld et al. 1984a). Direct charge recombination from $Q_{B}^{-}$to $P^{+}$is negligible, with recombination from the secondary quinone site, $P^{+} Q_{A} Q_{B}^{-} \rightarrow$ $P Q_{A} Q_{B}$, finally occurring through the primary quinone in $\sim 1 \mathrm{~s}$ in the dark-adapted state (Labahn et al. 1994).

When considering experiments performed under steadystate illumination with intensity $I_{\text {exp }}$, the effective forward ET rate is affected by the frequency of photoexcitation, which is dependent upon the light flux (intensity) and the oscillator strength of the chromophores. The absorption band of the primary photoelectron donor $P\left(\lambda_{\max }=865 \mathrm{~nm}\right)$ bleaches upon photoexcitation, signaling the creation of the radical pair $P^{+} Q_{A} Q_{B}^{-}$and providing a convenient method for monitoring the charge separation, electron transfer, and charge recombination kinetics (Clayton 1965). As is well known, appreciable amounts of the quinones at the $Q_{B}$ site may be lost during the RC isolation procedure (Shinkarev and Wraight 1997). The overall transmittance recovery kinetics following pulsed photoexcitation reflects the heterogeneity of the sample and is usually analyzed by fitting with a biexponential decay function with the components describing charge recombination in two types of RCs-those with no quinone (fast component) and those containing a quinone (slow component) in the $Q_{B}$ site:

$\Delta T_{865}(t)=C_{0}+C_{A} \exp \left(-\frac{t}{\tau_{A}}\right)+C_{B} \exp \left(-\frac{t}{\tau_{B}}\right)$,

where $\tau_{A}, C_{A}$ and $\tau_{B}, C_{B}$ are the lifetime and amplitude of the fast and slow recombination components, respectively, and $C_{0}$ is a constant. The amplitudes $C_{A}$ and $C_{B}$ should be replaced with their normalized equivalents $C_{1}$ and $C_{2}$ for the normalized transmittance recovery kinetics.

Our previous studies have shown that primary-donor dark recovery kinetics, upon cessation of continuous wave $(\mathrm{CW})$ photoexcitation, depends strongly upon the photoexcitation intensity and duration (Goushcha et al. 2003; Goushcha et al. 2004). In the analysis of experimental results of RC equilibration kinetics during various illumination conditions, it has been necessary to relate the experimentally measured values of light intensity $I_{\exp }$ with corresponding theoretical values $I$, the frequency of photoexcitation of a single RC per unit time. It is usually assumed that

$I=\alpha \cdot I_{\exp }$,

in which, for electric dipole transitions, the parameter $\alpha$ can be thought of as a function of the matrix element of the 
photoelectron donor $(P)$ dipole transitions from the ground to photoexcited state and the overlapping matrix element for the vibrational sub-states of the corresponding ground and excited electronic states of $P$ (see Appendix). A method for determination of the parameter $\alpha$, relating actinic light intensity values in units of power/(unit area) to values in units of reciprocal seconds, is presented in this work for isolated and membrane-bound RCs. This method uses an approach that applies the classical Bouguer-Lambert-Beer (BLB) formalism and is shown to give reasonably good results when scattering effects are present.

\section{Materials and methods}

\section{Samples}

Isolated RCs from the photosynthetic bacteria Rhodobacter $(R b$.) sphaeroides strain R26 and membrane-bound RCs from the antennae-free strain RC01 were used for this study. Isolated RCs were prepared with either LDAO (lauryl-N,N,-dimethylamine-N-oxide) or Triton X-100 detergent buffer solution. RC concentrations were determined from their absorption using the molar absorption coefficient of $2.88 \times 10^{5} \mathrm{M}^{-1} \mathrm{~cm}^{-1}$ at $802 \mathrm{~nm}$ (Straley et al. 1973) and ranged from 1 to $2 \mu \mathrm{M}$. The absorbance ratio $\frac{A_{280}}{A_{800}}$ for isolated RCs ranged from 1.25 to 1.35 , demonstrating good purity.

\section{LDAO sample}

Isolated RCs were prepared from photosynthetic membranes using the detergent LDAO according to the procedure described previously (Feher and Okamura 1978). Following purification on a column of oxiapatite, RCs were suspended in a solution of $10 \mathrm{mM}$ Tris- $\mathrm{HCl}(\mathrm{pH}=8.0)$, $1 \mathrm{mM}$ EDTA, and $0.025 \%$ LDAO. The RC suspension was then dialyzed against an excess of the detergent LDAO (0.05\%, pH 7.5) according to conventional methods. Quinone reconstitution was carried out to increase the $Q_{B}$ site occupancy by adding the ubiquinone isoprene homologue ubiquinone-4 (Q-4), as opposed to the RCs naturally occurring 10 isoprenoid unit ubiquinone-10 (Q-10), in a concentration $\sim 5-10$ times that of the RC concentration.

\section{Triton $X$-100 sample}

Isolated RCs were prepared from photosynthetic membranes using the detergent LDAO and a poly-histidine tag for rapid isolation according to the procedure described previously (Feher and Okamura 1978; Lin et al. 2001; Goldsmith and Boxer 1996). Following purification on a column of oxiapatite, RCs were suspended in $10 \mathrm{mM}$
Tris-HCl buffer with $0.05 \%$ LDAO, pH 7.5. The RC suspension was then dialyzed against an excess of the detergent Triton $\mathrm{X}-100(0.05 \%, \mathrm{pH} \quad 7.5)$ according to conventional methods. No quinone reconstitution procedure was used for this sample.

\section{Membrane-bound RCs}

Membrane-bound RCs from the Rhodobacter sphaeroides strain RCO1 were used. This strain lacks both LH1 and LH2 antenna complexes and is a photosynthetically competent strain that may contain active cytochrome $b_{1}$ complexes. The ratio of $\mathrm{RCs}$ to $\mathrm{bc}_{1}$ complexes was approximately $3: 1$ and the cytochrome $c_{2}$ was depleted in these membranes (Jones et al. 1992). Concentrated membranes were diluted with a buffer solution of Tris- $\mathrm{HCl}$ (20 mM tris-hydroxy-methyl-amine-methane) with a pH of 8.0 .

Membrane solution was filtered using 0.45-micron syringe filters ( $\mu$ Star, Corning Costar Corporation). Although cytochrome $c_{2}$ was depleted from the membrane samples, thus preventing reduction of oxidized $P^{+}$, the electron inhibitors myxothiazol (Sigma) and antimycin A (Sigma) were used to disable the $\mathrm{bc}_{1}$-complex function by preventing critical redox reactions occurring in the complex (Crofts 2004) and preventing reduction of any water soluble cytochrome $c_{2}$. Myxothiazol and antimycin A were dissolved in a small amount of ethanol and added in 5-fold excess of RC concentration to the membrane samples, with the total ethanol in each sample not exceeding $\sim 1 \%$. The three samples-one of pure membranes, one containing membranes with myxothiazol, and the third one containing membranes with both myxothiazol and antimycin A-were left overnight at $4^{\circ} \mathrm{C}$ for subsequent use in experiments at room temperature. $\mathrm{RC}$ concentrations in the membrane samples was ca. $1 \mu \mathrm{M}$. The similar kinetics for the membrane samples with and without the cytochrome $b_{1}$ inhibitors antimycin A and myxothiazol evidenced that the amount of cytochromes in these samples was negligible (see Results and Discussion below). Light scattering in the membrane samples was characterized as described below.

Photobleaching kinetics experimental methods

Transient absorption experiments were carried out using the optical setup described here and depicted schematically in Fig. 1. Samples in a 1-cm quartz cuvette were placed in a holder inside a black-anodized, aluminum sample compartment having entrance and exit apertures for the monitoring and excitation light. A quartz tungsten-halogen lamp (Sciencetech Inc. model TH2 housing and model 500-200/ Q controller) coupled to a monochromator was used for the source of measuring (monitoring) light at $865 \mathrm{~nm}$ (slit 
Fig. 1 Simplified block schematic of the experimental setup. See text for details. $F$ filter, $L$ lens, $D$ diaphragm, $C$ cuvette, $P$ periscope, $P D$ photodetector, $Q T H$ quartz tungsten halogen

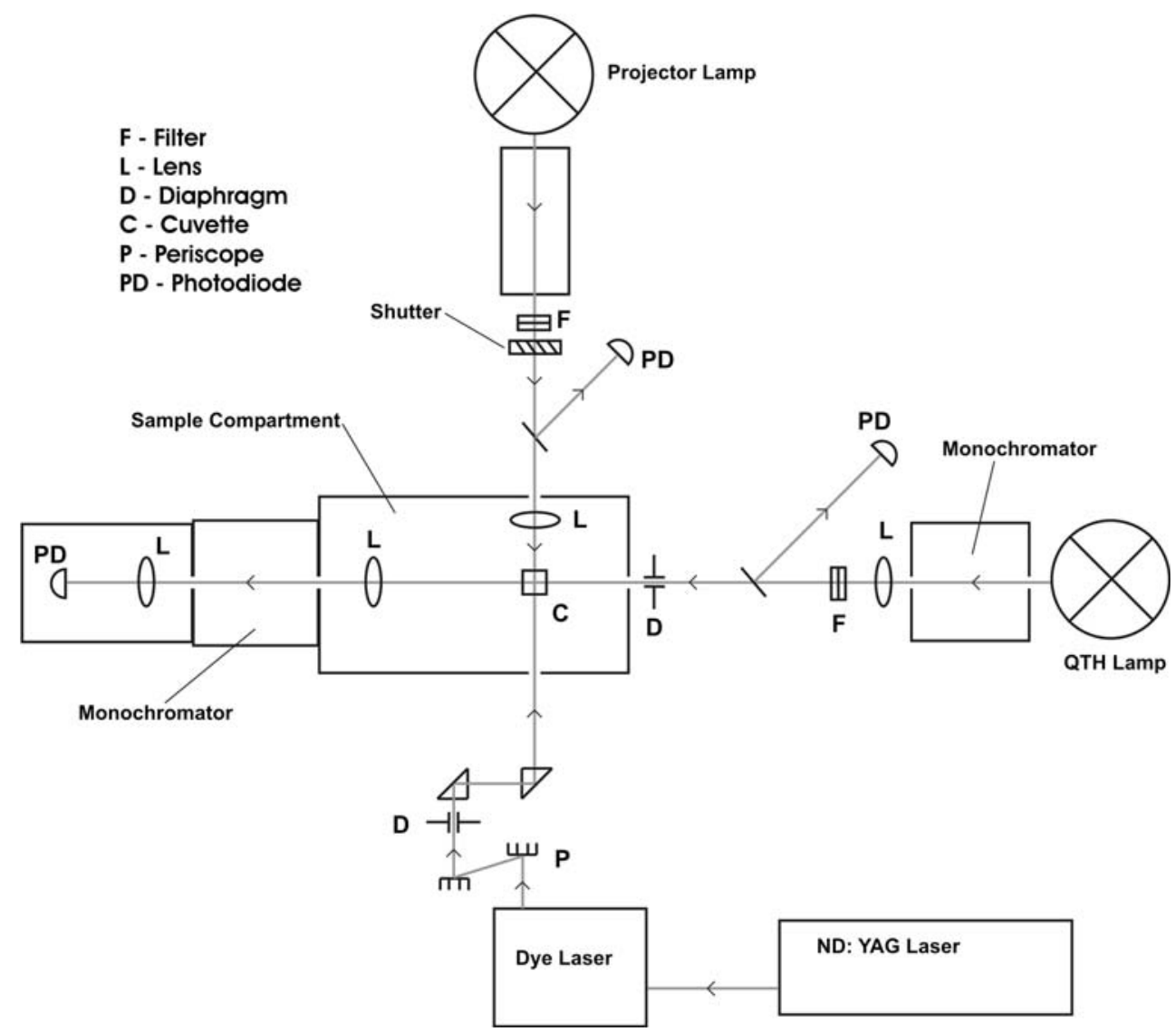

bandwidth $=20 \mathrm{~nm}$ ). The monitoring light was filtered with a red cutoff filter RG-630 (Schott) and neutral density filters were used for the intensity control. An iris diaphragm was placed in the monitoring beam path to control the beam diameter (usually $<3 \mathrm{~mm}$ ). The monitoring light intensity was $<5 \mu \mathrm{W} / \mathrm{cm}^{2}$. After passing through the sample the light was focused onto the entrance slit of a second monochromator set at $\lambda=865 \mathrm{~nm}$ to eliminate ambient and scattered actinic light.

CW white excitation light was supplied by a tungstenhalogen lamp and then filtered with a $10-\mathrm{cm}$ path water filter and a cutoff filter OG-550 (Schott), resulting in excitation wavelengths within the range $\lambda_{\mathrm{cw}}=600-$ $900 \mathrm{~nm}$. An electronic shutter (Melles-Griot) was placed in the $\mathrm{CW}$ beam path to switch the light on and off. A set of neutral density filters was used to vary the light intensity on the sample up to $I_{\exp } \sim 20 \mathrm{~mW} / \mathrm{cm}^{2}$. The intensity of the $\mathrm{CW}$ illumination incident upon the RC samples was measured with an Ophir Nova meter in conjunction with a Nova model 3A-P-SH thermopile head. The second harmonic from a Quanta-Ray DCR-3 Pulsed Nd:YAG Laser (Spectra-Physics) was used to pump a Quanta-Ray PDL-2 dye laser that served as the source of the actinic light pulses. The dye laser was tuned to $605 \mathrm{~nm}$ using Rhodamine 640 as the dye. The pulse energy at $605 \mathrm{~nm}$ was $\sim 50 \mathrm{~mJ}$, and care was taken to provide a uniform excitation across the surface of the sample (ca. $1 \mathrm{~cm}^{2}$ excitation area). The $\mathrm{CW}$ and pulsed excitation of the sample were at a $90^{\circ}$ angle to the monitoring beam.

The intensities of the monitoring light before entering and after exiting the sample chamber, and the intensity of the $\mathrm{CW}$ actinic light, were monitored simultaneously with photodiodes coupled to wide bandwidth preamplifiers to check for any instability in the light sources in addition to monitoring the sample absorbance. The signals from the preamplifiers were acquired with a 12-bit plug-in dataacquisition board (Keithley DAS-1801 ST-DA) in conjunction with a Pentium based PC. The digital outputs of this board triggered the shutter and the laser pulses.

Theoretical modeling

Rhodobacter sphaeroides RCs can be considered as a two level system of the charge-neutral $(D A)$ and the chargeseparated $\left(D^{+} A^{-}\right)$states with the charge recombination rate constant $k_{\text {rec }}$ equal either to the rate constant $k_{A}=k_{A P}$ $\approx 10 \mathrm{~s}^{-1}$ for the radical pair $D^{+} Q_{A}^{-}$of $Q_{B}$-lacking RCs, or to $k_{B} \approx k_{A P} \frac{k_{B A}}{k_{A B}} \sim 1 \mathrm{~s}^{-1}$ for $Q_{B}$-containing RCs (Labahn et al. 1994; Kleinfeld et al. 1984b). The normalized, time dependant populations of the charge neutral $\rho(t, D)$ and charge 
separated $\rho(t, A)$ states at time t satisfy the simple coupled differential rate equations

$$
\begin{aligned}
& \frac{\partial \rho(t, D)}{\partial t}=-I \rho(t, D)+k_{\mathrm{rec}} \rho(t, A) \\
& \frac{\partial \rho(t, A)}{\partial t}=I \rho(t, D)-k_{\mathrm{rec}} \rho(t, A)
\end{aligned}
$$

The solution of Eq. 3 is

$$
\begin{aligned}
\rho(t, D) & =1-\rho(t, A) \\
& =\rho_{I}(\infty, D)+\left[\rho(0, D)-\rho_{I}(\infty, D)\right] \exp (-\kappa t),
\end{aligned}
$$

where $\kappa=I+k_{\text {rec }}$, and the solutions for the normalized populations take hyperbolic forms with respect to $I$ and $k_{\text {rec }}$ when the system reaches steady-state, $t \rightarrow \infty$ (Abgaryan et al. 1998; Goushcha et al. 2000).

\section{Method 1}

Assuming that the transmittance is zero at time $t=0$ and $I_{\exp }=0$, the exact solution for the RC bleaching kinetics for a sample with a mixture of $Q_{B}$-depleted and $Q_{B}$-active RCs following a sudden increase of the light intensity to level $I_{\exp }>0$ is described by the expression

$$
\begin{aligned}
T_{865}\left(I_{\exp }, t\right)= & C_{A} \frac{\alpha \cdot I_{\exp }}{\alpha \cdot I_{\exp }+k_{A}}\left[1-\exp \left(-t\left(\alpha \cdot I_{\exp }+k_{A}\right)\right)\right] \\
& +C_{B} \frac{\alpha \cdot I_{\exp }}{\alpha \cdot I_{\exp }+k_{B}}\left[1-\exp \left(-t\left(\alpha \cdot I_{\exp }+k_{B}\right)\right)\right],
\end{aligned}
$$

where $C_{A}$ and $C_{B}$ are constants with the arbitrary units used for the experimentally measured transmittance levels, the experimental excitation intensity is $I_{\text {exp }}$, and all other parameters are defined above. In this study, the sample transmittance was always measured at $865 \mathrm{~nm}$ and this is denoted by a subscript on $T$ in Eq. 5. When normalized, the amplitudes of $C_{A}$ and $C_{B}$ give the relative amounts of $Q_{B^{-}}$ depleted and $Q_{B}$-active RCs in the sample. The ratios in each term of Eq. 5 gives the extent that each RC sample component contributes to the overall steady state saturation level.

\section{Method 2}

A second method of analysis uses a single effective lifetime for the redox state of the whole system, regardless of whether it is a single component system or a multiple component system. The effective rate constant of electronic equilibration, $\tau_{e l}^{-1}$, is

$\tau_{e l}^{-1}=I+k_{\mathrm{rec}}^{\prime}=I+\left[\frac{C_{A}}{k_{A}}+\frac{C_{B}}{k_{B}}\right]^{-1}$,

and the effective charge recombination rate, or rate constant for electron transfer back to the bacteriochlorophyll dimmer (donor), $k_{\mathrm{rec}}^{\prime}=\tau_{d}^{-1}$, is given by the term in brackets. The overall bleaching kinetics then follows the relation:

$T_{865}(I, t)=C \frac{\alpha \cdot I_{\exp }}{\alpha \cdot I_{\exp }+k_{\text {rec }}^{\prime}}\left(1-\exp \left[-t\left(\alpha \cdot I_{\exp }+\tau_{d}^{-1}\right)\right]\right)$.

The factor $C$ in Eq. 7 relates the measured transmittance in arbitrary units to the dimensionless theoretical quantity. The effective charge recombination lifetime, $\tau_{d}=\left(k_{\mathrm{rec}}^{\prime}\right)^{-1}$, can also be considered as an "average survival time" of the charge separated state(s) with respect to the donor (Agmon and Hopfield 1983; Abgaryan et al. 1998) in cases where charge recombination becomes multiexponential. It has been shown previously (Abgaryan et al. 1998; Goushcha et al. 2000) that the recombination kinetics for a complex RC system can be described using such a single effective decay parameter. For the general case of a system with a fixed structure and a finite number of localized electron states, the value of this effective decay parameter depends only on structural organization and not upon the actinic light intensity, with changes in this effective decay parameter value attributed to structural changes within the RC system.

Method 2 describes a mixture of $Q_{B}$-active and $Q_{B^{-}}$ depleted RCs as a single homogeneous donor-acceptor system with a single effective recombination rate and is not independent of the more rigorous Method 1. Both methods apply directly to isolated RCs samples, yet Method 2 can be used in situations where Method 1 may be more difficult to apply. The above descriptions can be applied, with some precautions, to membrane-bound RCs samples, in which multiple scattering effects occur (Goushcha et al. 2004). We will use Method 2 to make an approximate estimation of the excitation parameters for membrane samples.

\section{Results}

Rate constants obtained from flash activated kinetics

The charge recombination kinetics following a single actinic flash applied to dark-adapted samples are analyzed with the two-exponential decay function given by Eq. 1 . Representative fitting results for isolated RCs are listed in Table 1. The relative amplitudes and time constants obtained from these results are used to calculate $k_{\text {rec }}^{\prime}$ and are also shown in Table 1. The single exponential decay lifetimes of isolated RCs and membranes after applying a single actinic flash are (assuming no structural changes under our excitation conditions) $\tau_{s}=0.84 \mathrm{~s}$ for RCs with LDAO, $\tau_{s}=0.20 \mathrm{~s}$ for RCs with Triton X-100, $\tau_{s}=4.59 \mathrm{~s}$ for membranes, $\tau_{s}=4.69 \mathrm{~s}$ for membranes with myxothiazol, and $\tau_{s}=4.33 \mathrm{~s}$ for membranes with 
Table 1 The fitting results for the single flash-activated, dark recovery kinetics of isolated RC samples

\begin{tabular}{llllll}
\hline Sample & $C_{1}$ & $\tau_{A}, \mathrm{~s}$ & $C_{2}$ & $\tau_{B}, \mathrm{~s}$ & $k_{\mathrm{rec}}^{\prime}, \mathrm{s}^{-1}$ \\
\hline LDAO & 0.36 & $0.28(3.57)$ & 0.64 & $1.16(0.86)$ & 1.18 \\
Triton X-100 & 0.71 & $0.112(9.1)$ & 0.29 & $0.45(2.23)$ & 4.81 \\
\hline
\end{tabular}

$C_{1}$ and $C_{2}$ are the normalized, relative amounts of the RCs that are $Q_{B}$-depleted and $Q_{B}$-occupied. $\tau_{A}$ and $\tau_{B}$ are the time constants for charge recombination. The values in parenthesis next to the $\tau_{A}$ and $\tau_{B}$ values denote the inverse of the time constants in $\mathrm{s}^{-1} . k_{\mathrm{rec}}^{\prime}$ is the effective single charge recombination constant determined by using the single flash data $\left(C_{1}, C_{2}, \tau_{A}\right.$, and $\left.\tau_{B}\right)$ with Eq. 6

myxothiazol and antimycin A (see Samples in Materials and methods section). These single exponential decay lifetimes can be compared with the values of $\tau_{d}=\left(k_{\text {rec }}^{\prime}\right)^{-1}$ given in Table 1 for isolated RCs.

RC bleaching kinetics and resulting fits

Figure 2 shows typical results of absorbance bleaching kinetics for RCs with Triton X-100 following a sudden increase of the actinic light intensity, starting in the dark, to nine different excitation levels, $I_{\text {exp }}$. The smooth lines show the results of a global fitting using all nine bleaching curves for each excitation level $I_{\text {exp. Note that both analysis }}$ methods (Method 1 and Method 2) provide excellent fitting results. For fitting experimental results to each model, the light intensity parameters are held fixed for each curve and all other parameters are shared and allowed to float. In the analysis, it is assumed that, within the 2-second time interval of applied illumination, the electron transfer rate constants do not change by light induced structural changes (Goushcha et al. 2003; Goushcha et al. 2004). Figure 3 shows typical bleaching kinetics for RCs with LDAO, and Fig. 4 represents typical bleaching kinetics for membranebound RCs. The fitted curves in Fig. 4 for the membranebound RCs are obtained using analysis Method 2. The measured and fitted bleaching kinetics for several samples of isolated RCs with Triton X-100 and LDAO, and for membrane-bound RCs, are summarized in Table 2.

The light intensity values used for $I_{\text {exp }}$ are the estimated excitation intensities at the middle of the sample cuvette and are determined separately for each sample trial. First, the excitation intensity at the incident surface of the cuvette is measured. The excitation intensity at the middle of the cuvette is then estimated by applying the BLB law, using the extinction coefficient at $802 \mathrm{~nm}$, and the measured absorbance values at this wavelength to estimate the excitation intensity at the middle of the cuvette (see Table 3). Since the monitoring beam diameter is less than $3 \mathrm{~mm}$, we assume that the $I_{\exp }$ value is constant across the whole monitoring beam in the middle of the cuvette. This
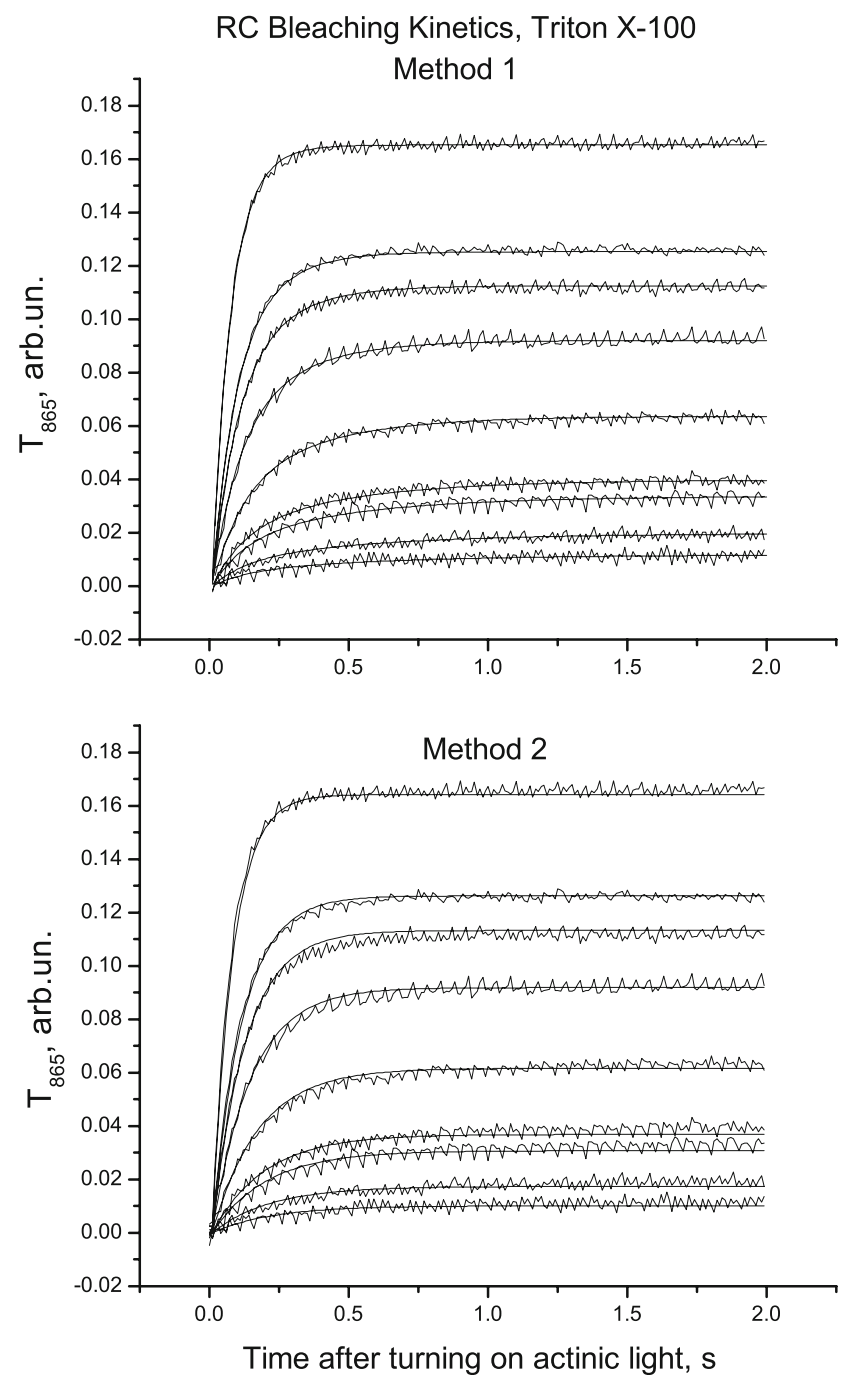

Fig. 2 Bleaching kinetics of Triton X-100 isolated RCs after turning on $\mathrm{CW}$ illumination for a 2 -second time interval. The transmittance at a wavelength of $865 \mathrm{~nm}, \mathbf{T}_{865}$, versus time is shown. The smooth line shows the results of fitting using Method 1 (top graph) and Method 2 (bottom graph)

assumption may not be completely valid for the sample with membranes due to scattering effects. Because of this, scattering effects within membrane bound samples were investigated further.

The type and amount of scattering in the membrane samples was estimated by fitting the absorption curve of a membrane sample to the sum of a scaled, previously measured isolated RC absorption spectrum and the scattering formula $A_{\text {scatter }}=C_{S} \cdot \lambda^{K_{S}}$, where $C_{S}$ is a constant and $K_{S}$ characterizes the scattering. For small particles with respect to the wavelength, $K_{S}=-4$ and is representative of Rayleigh scattering. Values of $K_{S}$ above -4 and approaching zero are more characteristic of Mie scattering (Cavatorta et al. 1986; Hudson 1969). Figure 5 shows the resulting least squares fit of the membrane absorption spectrum and 

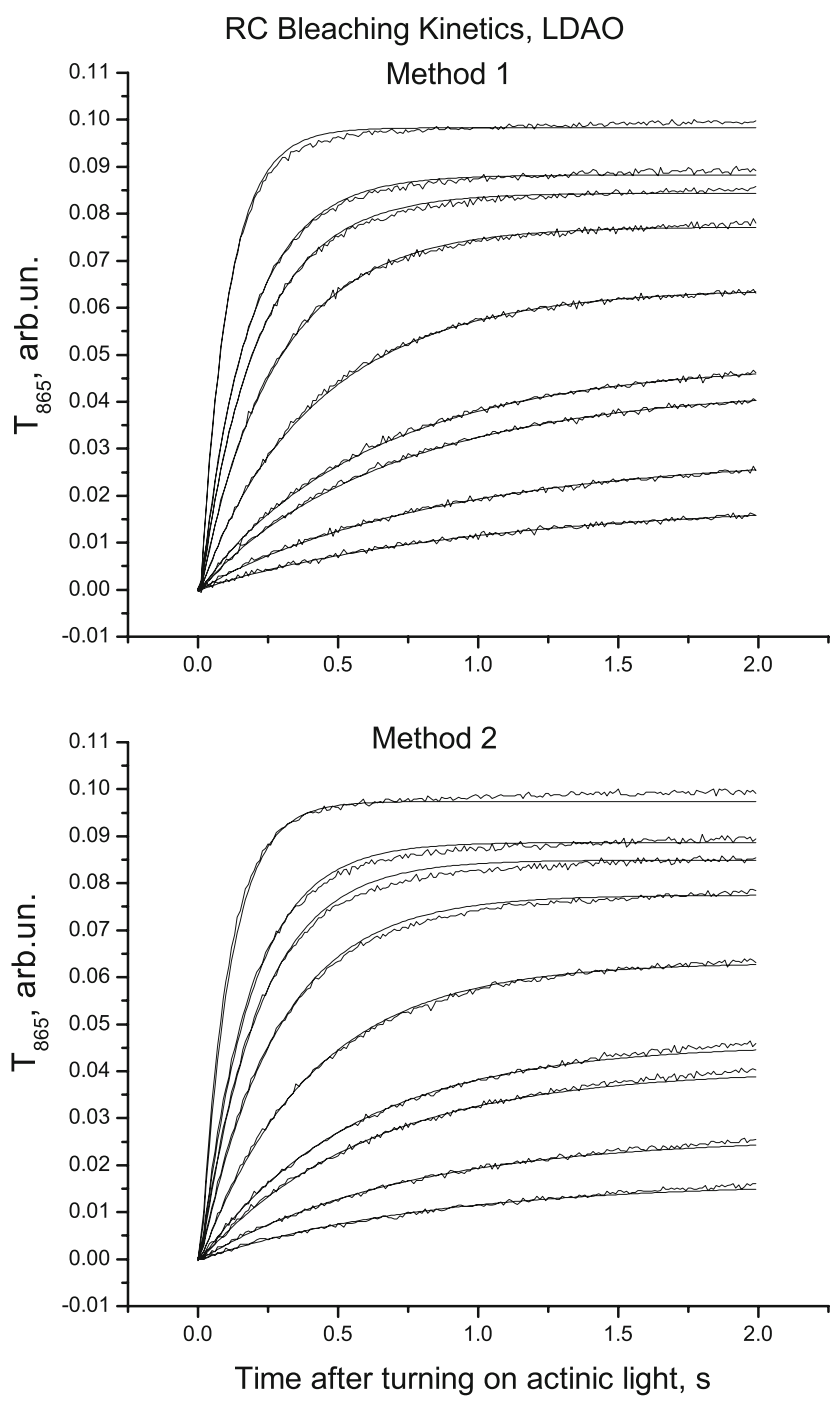

Fig. 3 Bleaching kinetics of LDAO isolated RCs after turning on $\mathrm{CW}$ illumination for a 2 -second time interval. The transmittance at a wavelength of $865 \mathrm{~nm}, \mathbf{T}_{865}$, versus time is shown. The smooth line shows the results of fitting using Method 1 (top graph) and Method 2 (bottom graph)

the corresponding curve for $A_{\text {scatter }}$. From the analysis, the values $\log \left[C_{S}\right]=8 \pm 0.05$ and $K_{S}=-2.95 \pm 0.02$ were obtained. The value of $K_{S}$ indicates that the scattering is more like that of Mie scattering, or Rayleigh-Debye-Gans scattering, in which case the dimension of the scattering particle was large and could not be treated as a single dipole (Cavatorta et al. 1986; Hudson 1969). The absorption at $802 \mathrm{~nm}$, after subtracting the scatter curve $A_{\text {scatter }}$ from the membrane absorption, was used to determine the concentrations to be $\sim 1 \mu \mathrm{M}$. This analysis, however, does not address possible multiple scattering effects fully, which were found to play a large role in RC photoexcitation dynamics (Goushcha et al. 2004) and are discussed further below.

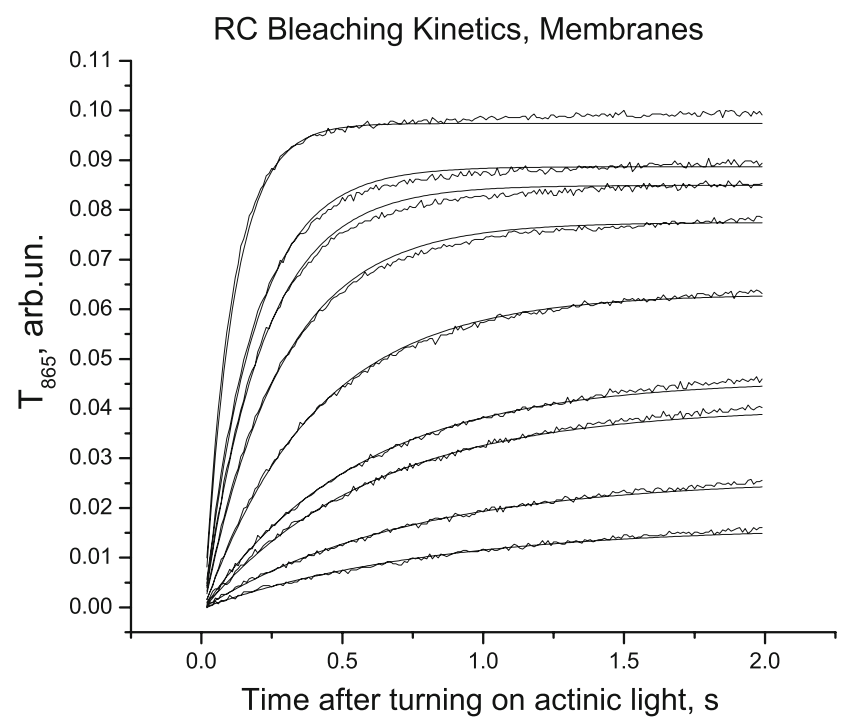

Fig. 4 Bleaching kinetics of membrane bound RCs after turning on $\mathrm{CW}$ illumination for a 2 -second time interval. The transmittance at a wavelength of $865 \mathrm{~nm}, \mathbf{T}_{865}$, versus time is shown. The smooth line shows the results of fitting using Method 2

Figure 6 shows a simplified schematic of the cuvette compartment. The monitoring beam propagates along the $x$-axis, and $\mathrm{CW}$ excitation is applied along the $y$-axis. Since the scattering is pronounced in membrane samples, the actual $\mathrm{CW}$ excitation beam intensity in the middle of the cuvette (the hatched region of the cuvette in Fig. 6) is significantly smaller than on the surface of the cuvette, and this is also inferred from the absorption spectrum. Moreover, this light intensity changes along the $y$-axis within the width of the monitoring beam, producing a noticeably non-uniform excitation profile. Comparison of absorption measurements at the $802 \mathrm{~nm}$ absorption band of membrane-bound RCs in $1 \mathrm{~cm}$ and $1 \mathrm{~mm}$ path length cuvettes also reveals such attenuations. However, we have previously shown that for a fixed $\mathrm{CW}$ excitation intensity the bleaching kinetics is significantly increased with increasing beam diameter, indicating that multiple scattering effects are also in play and can compete with the attenuation effects (Goushcha et al. 2004). For membranebound RCs, using a $1 \mathrm{~cm}$ path length cuvette, the effective excitation intensity for the membrane-bound RCs is shown to be $\sim 10$ times that of the incident excitation intensity due to the scattering inside the sample. Due to the same multiple scattering effects, the overall beam attenuation in the middle of the cuvette with membranes is significantly larger than what is expected due to simple absorption governed by the BLB law. These two competing effects, beam attenuation and multiple scattering, complicate calculations for the membrane-bound RCs, allowing only a qualitative analysis of the bleaching kinetics in those samples. 
Table 2 Summary of the light intensity parameter and effective recombination rate constant values for isolated and membrane-bound RCs

\begin{tabular}{llllllll}
\hline Sample & $\begin{array}{l}\alpha \mathrm{ml} \\
\mathrm{mW}^{-1} \mathrm{~cm}^{2} \mathrm{~s}^{-1}\end{array}$ & $\begin{array}{l}\alpha \mathrm{m} 2 \\
\mathrm{~mW}^{-1} \mathrm{~cm}^{2} \mathrm{~s}^{-1}\end{array}$ & $\begin{array}{l}k_{\text {rec }}^{\prime} \mathrm{s}^{-1} \\
\text { (uncertainty) }\end{array}$ & $\begin{array}{l}k_{A}, \mathrm{~s}^{-1} \\
\text { (uncertainty) }\end{array}$ & $\begin{array}{l}k_{B}, \mathrm{~s}^{-1} \\
\text { (uncertainty) }\end{array}$ & $\begin{array}{l}C_{A} \text { arb. un. } \\
\text { (uncertainty) }\end{array}$ & $\begin{array}{l}C_{B} \text { arb. un. } \\
\text { (uncertainty) }\end{array}$ \\
\hline LDAO & $0.8180(0.0004)$ & $0.8171(0.0006)$ & $1.056(0.001)$ & $8.29(0.24)$ & $0.758(0.005)$ & $0.0280[0.23](0.0002)$ & $0.0914[0.77](0.0004)$ \\
Triton X-100 & $0.965(0.001)$ & $0.979(0.002)$ & $4.491(0.008)$ & $7.92(0.12)$ & $1.49(0.05)$ & $0.217[0.78](0.002)$ & $0.059[0.22](0.002)$ \\
Membranes & $8.72(0.02)$ & $6.30(0.02)$ & $0.817(0.005)$ & $18.36(0.89)$ & $0.22(0.01)$ & $0.046(0.54)(0.001)$ & $0.0386(0.46)(0.0003)$
\end{tabular}

$\alpha m l$ and $\alpha m 2$ are the light intensity conversion parameters obtained experimentally using Method 1 and Method 2, respectively. $k_{\text {rec }}^{\prime}$ is the charge recombination rate obtained using analysis Method 2, and $k_{A}$ and $k_{A}$ are the charge recombination rates obtained using analysis Method $1 . C_{A}$ and $C_{B}$ are the relative proportions of $Q_{B}$-depleted and $Q_{B}$-enriched RCs in the sample, respectively. The values in square brackets next to $C_{A}$ and $C_{B}$ are the normalized portions of $Q_{B}$-depleted and $Q_{B}$-active RCs. The values in parenthesis underneath the measured values are the uncertainties for those measurements

Table 3 Photoexcitation intensities measured at the surface of incidence and estimated at the middle of the sample cuvette for isolated and membrane-bound RCs

\begin{tabular}{llll}
\hline Parameter & $\begin{array}{l}I_{\text {exp }} \text { at the } \\
\text { surface of the } \\
\text { cuvette, } \\
\text { mW cm }\end{array}$ & $\begin{array}{l}\text { Estimated } I_{\text {exp }} \text { in } \\
\text { the middle of the } \\
\text { cuvette with } \\
\text { isolated RCs, } \\
\mathrm{mW} \mathrm{cm}^{-2}\end{array}$ & $\begin{array}{l}\text { Estimated } I_{\text {exp }} \text { in the } \\
\text { middle of the } \\
\text { cuvette with } \\
\text { membrane-bound } \\
\text { RCs, mW cm }\end{array}$ \\
\hline$I_{\text {exp_1 }}$ & 18.07 & 9.16 & 0.92 \\
$I_{\text {exp_2 }}$ & 9.51 & 4.82 & 0.48 \\
$I_{\text {exp_3 }}$ & 7.70 & 3.91 & 0.39 \\
$I_{\text {exp_4 }}$ & 5.38 & 2.76 & 0.27 \\
$I_{\text {exp_5 }}$ & 3.02 & 1.52 & 0.15 \\
$I_{\text {exp_6 }}$ & 1.59 & 0.81 & 0.08 \\
$I_{\text {exp_7 }}$ & 1.29 & 0.65 & 0.07 \\
$I_{\text {exp_8 }}$ & 0.69 & 0.35 & 0.04 \\
$I_{\text {exp_9 }}$ & 0.39 & 0.2 & 0.02 \\
\hline
\end{tabular}

\section{Discussion}

For the case of Triton X-100 (see Fig. 2 and Table 2), using light intensities given in units of $\mathrm{mW} / \mathrm{cm}^{2}$, a representative value of the light intensity parameter $\alpha$ equal to $0.97\left(\mathrm{~s}^{-1} \mathrm{~cm}^{2} / \mathrm{mW}\right)$ is obtained using Method 1 . The rate constants $k_{A}=7.92 \mathrm{~s}^{-1}$ and $k_{B}=1.49 \mathrm{~s}^{-1}$ obtained from the analysis of the bleaching kinetics agree well with the recombination rate constant values from the literature, yet they are slightly different from the corresponding values of 9.1 and $2.23 \mathrm{~s}^{-1}$ obtained from the single flash dark recovery experiments (shown in Table 1). The ratio of $0.78-0.22$ of $Q_{B}$-depleted to $Q_{B}$-active RCs is in reasonable agreement with the ratio obtained from single flash dark recovery kinetics $(0.71-0.29)$. The $\alpha$ value of $0.98 \mathrm{~s}^{-1} \mathrm{~cm}^{2} / \mathrm{mW}$ obtained using Method 2 is essentially equivalent to that obtained using Method 1 . The effective recombination rate constant $k_{\text {rec }}^{\prime}$, obtained from Method 2 is $4.49 \mathrm{~s}^{-1}$. Applying this effective recombination rate along

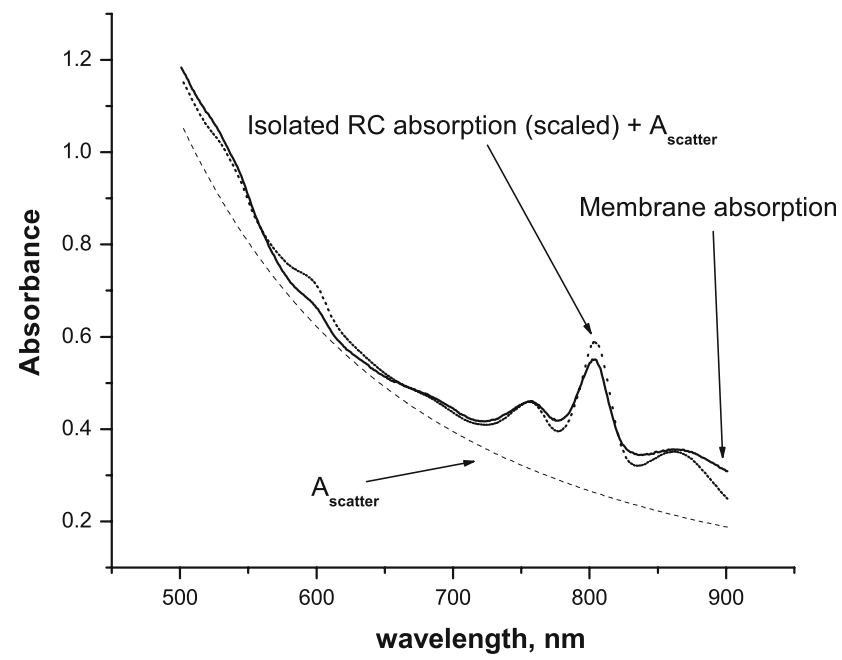

Fig. 5 Effects of multiple light scattering in membrane-bound RCs. Solid line is membrane absorption curve. Dotted line is the scaled isolated RC spectrum $+A_{\text {scatter. }}$ The dashed line below these curves is the contribution due to scattering, $A_{\text {scatter, }}$ in the absorption spectrum

with the rate constants from the single flash dark recovery kinetics $\left(k_{A} \approx 9.1 \mathrm{~s}^{-1} k_{B} \approx 2.23 \mathrm{~s}^{-1}\right)$ to $k_{\mathrm{rec}}^{\prime}$ in Eq. 6 results in the portions of $Q_{B}$-depleted and $Q_{B}$-active RCs being 0.67 and 0.33 , respectively, which is in fair agreement with the portions determined using Method 1 (see Table 2).

For the LDAO sample of Fig. 3 (see fitting parameters in Tables 2), the $\alpha$ parameter values obtained with Methods 1 and 2 are the same and equal to $\approx 0.82 \mathrm{~cm}^{2} / \mathrm{mW} \mathrm{s}$. The $Q_{B}$-depleted to $Q_{B^{-}}$active ratios are $0.23-0.77$ using Method 1, and 0.36-0.64 from the analysis of the single flash-activated dark decay kinetics. The $k_{\text {rec }}^{\prime}$ value obtained using Method 2, $1.06 \mathrm{~s}^{-1}$, is close to the value of $1.18 \mathrm{~s}^{-1}$ calculated from the single flash dark recovery kinetics using $k_{\text {rec }}^{\prime}$ from Eq. 6 .

Although neither modeling scheme worked perfectly well for the membrane-bound RCs, Method 2 produced 


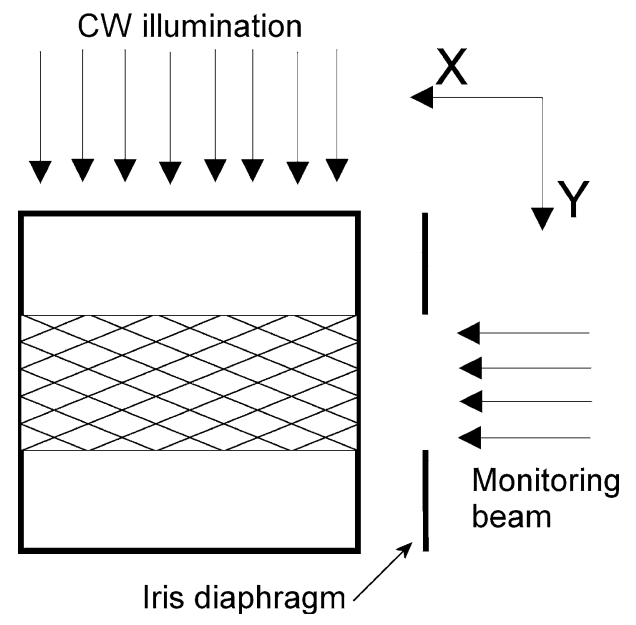

Fig. 6 Simplified schematic of the cuvette compartment with the CW illumination and monitoring (testing light) configuration. The entire RCs sample is exposed to the $\mathrm{CW}$ illumination along the $y$-axis. The monitoring beam along the $x$-axis illuminates only a $\sim 3 \mathrm{~mm}$ diameter portion of the $\mathrm{CW}$ illuminated sample due to blocking by the iris diaphragm, resulting in only the hatched region being monitored for the transmittance measurements

reasonably good results. Complications may arise with the membrane samples due to strong light scattering, which simultaneously produces two competitive effects-a pronounced decrease in the light intensity along the excitation beam (scattering attenuation) and an increased photoexcitation intensity due to multiple scattering. The light parameter $\alpha$ obtained for the sample with membranes is approximately 10 times bigger than that for isolated RCs $\left(6.3 \mathrm{~mW}^{-1} \mathrm{~cm}^{2} \mathrm{~s}^{-1}\right.$ and higher for membrane-bound RCs), which is in agreement with our previous studies showing that the efficiency of photoexcitation increases significantly in membranes due to the light scattering effects (Goushcha et al. 2004). Our estimation of the excitation beam intensity in the middle of the cuvette with membranes is approximate and based upon previous studies using the same experimental setup (same sample concentration, same excitation and monitoring conditions, and same cuvette path length). The competition between scattering attenuation and increased excitation due to multiple scattering may vary depending upon path length, concentration, and excitation/ monitoring conditions for membrane samples. The relationship between $I$ and $I_{\exp }$ given in the Appendix, with the scaling parameter written in terms of the dipole transition matrix, supports the apparent relation between scattering attenuation and an increased effective photoexcitation.

From the above experimental results, the $k_{\text {rec }}^{\prime}$ value obtained for the membrane samples using Method 2 $\left(\approx 0.82 \mathrm{~s}^{-1}\right)$ is larger than the value of the recombination rate constant $\left(\approx 0.22 \mathrm{~s}^{-1}\right)$ measured using the single flash activated recovery kinetics. The difference should be attributed to two reasons: (1) uncertainty in determination of $I_{\text {exp }}$ using Method 2 due to scattering effects and (2) long lifetime of the charge separated state for membrane-bound RCs ( $\sim 3-5$ s, see Goushcha et al. 2004), which means that the 2-second exposure time in our experiments may not have been long enough for the correct determination of the rate constants. Taking these precautions into consideration, we used the measured value $k_{\text {rec }}^{\prime}=0.82 \mathrm{~s}^{-1}$ for determination of the scaling parameter $\alpha$ for membrane bound RCs.

The light parameter values $\alpha$ obtained in all the experiments with isolated RCs using the two different modeling methods are close to each other, ranging from $\sim 0.6$ to $\sim 1.1 \mathrm{~cm}^{2} / \mathrm{mW}$ s. Variations in sample conditions, such as $\mathrm{RC}$ concentration, detergent concentration, and $Q_{B}$ content are all possible reasons for the variation of the $\alpha$ parameters and rate constants observed for each experimental trial. Other reasons for the variations of these parameters are (1) the possibility of a changing $Q_{B}$ binding equilibrium and/or binding constant under continuous wave $(\mathrm{CW})$ illumination conditions and (2) possible light-induced structural changes that may be affecting charge transfer kinetics during the 2-s time interval used in the current studies to record the RC bleaching kinetics.

Although quinone reconstitution at the $Q_{B}$ site is only attempted for LDAO samples, full reconstitution is known to be difficult to obtain due to differences in the distribution and exchange of quinones between RC micelles, detergent micelles, and combined detergent-RC micelles (Shinkarev and Wraight 1997; Wraight 2004). These factors are reflected in the differences observed between our measured charge recombination lifetimes and the expected rates ( $\sim 10$ and $\sim 1 \mathrm{~s}^{-1}$ ) for all the isolated RC samples. It is not difficult to discern distinct fast and slow recombination rates for each isolated $\mathrm{RC}$ sample, with the amplitudes of a bi-exponential fit giving a good estimate of the $Q_{B}$-active and $Q_{B}$-depleted RCs portions. The time components of the charge recombination in such systems is influenced by the type and concentration of detergent used, the concentration of quinones, and the quinone binding constant at the $Q_{B}$ site. The amplitudes and time constants obtained from the single flash experiments are within the expected limits for our samples. It is not clear how much, if at all, the CW illumination affects the quinone binding constant or quinone distribution. The simple model used in this study does not account for such effects, which might be a cause for additional discrepancies between the values obtained from the different types of experiments (single flash decay kinetics and $\mathrm{CW}$ excitation bleaching kinetics).

A number of studies indicate that structural changes might occur in RCs during the photocycle event, including the uptake and release of protons to residues near key electron transfer sites and pathways (Wraight 2004). Our previous studies indicate that structural changes influence charge recombination kinetics on long time scales (Goushcha et al. 
2003; Goushcha et al. 2004). Such changes can also affect the electronic properties of the $\mathrm{RC}$ on shorter time scales, resulting in altered charge transfer kinetics. This may also be reason for discrepancies between the measured fast and slow charge recombination rates using the different methods.

The purification procedures of each sample are well established and it is highly unlikely that there is any effect upon the kinetics due to cytochrome $c_{2}$ remaining in the samples. The RC absorption spectrum is wide (600$900 \mathrm{~nm}$ ), yet only one absorption wavelength is monitored in this study in order to simplify the analysis. The $802 \mathrm{~nm}$ absorption band is the primary absorption band, and a more elaborate analysis over a wider spectral range may change our main results only slightly.

To the authors' knowledge, a detailed account of photoexcitation dynamics of RCs at room temperatures has not been previously reported on. We can refer to the recent work by Olenchuk et al. (2007), which describes the RCs equilibration dynamics at room temperatures; however, that work emphasizes the case of samples with rather strongly absorbing RC concentrations (or very low light photoexcitation levels) where the classical BLB formalism breaks down.

\section{Conclusion}

Detailed examination of the RCs equilibration kinetics under a sudden increase of the $\mathrm{CW}$ actinic light intensity from the dark to a particular steady-state level, $I_{\text {exp }}$, provides a tool for the correct and independent estimation of the light intensity parameter $\alpha$, the scaling factor to measure the molecule photoexcitation frequency. This parameter is very important for the correct theoretical modeling of the RCs dynamics, especially in determining the details of charge separation induced structural transitions in RCs. The models used here to describe the photobleaching kinetics and to determine the parameter $\alpha$ fits the experimental results very well and shows a reasonable agreement with the results of previous studies of electron transfer kinetics in isolated and membrane bound RCs. In other studies, the case of strong absorption that may cause saturation absorption was discussed theoretically and analyzed empirically for isolated RCs (Olenchuk et al. 2007). Our work more fully illustrates the methodology for the classical BLB formalism and emphasizes the analysis of experimental results when light scattering occurs, which allows for applying the BLB formalism to estimate the $\alpha$ factor.

Acknowledgments The authors would like to thank Dr. M.R. Jones for samples of the antenna-free membranes of $R b$. sphaeroides photosynthetic bacteria (strain RCO1), Dr. N. Woodbury for Triton X-100 isolated RCs, and Drs. G. Feher and M. Okamura for the LDAO isolated RCs that they each generously provided for these studies.
Open Access This article is distributed under the terms of the Creative Commons Attribution Noncommercial License which permits any noncommercial use, distribution, and reproduction in any medium, provided the original author(s) and source are credited.

\section{Appendix}

Equation 2 gives the rate of the forward reaction that creates the charge separated state $D^{+} A^{-}$which is determined by the donor photoexcitation, with a light frequency dependent transition probability $k_{\text {forward }}(\omega)$ that is given by

$$
\begin{aligned}
k_{\text {forward }}(\omega) \equiv I= & \frac{2 \pi}{\hbar}\left|\left\langle P_{1}|\vec{d} \vec{E}| P_{0}\right\rangle\right|^{2} \sum_{v} \sum_{v^{\prime}}\left|\left\langle v^{\prime} \mid v\right\rangle\right|^{2} \\
& \times \frac{\exp \left(-\omega_{v} / k_{B l t z} T\right)}{\sum_{v} \exp \left(-\omega_{v} / k_{B l t z} T\right)} \delta\left(\varepsilon_{10}+\omega_{v^{\prime} v}-\omega\right),
\end{aligned}
$$

in which $\left|\left\langle P_{1}|\vec{d} \vec{E}| P_{0}\right\rangle\right|$ is the dipole transition element for the donor transition from the ground electronic state $P_{0}$ to the excited state $P_{1}, \vec{d}$ is the dipole transition momentum, $\vec{E}=\vec{e} E$ is the electric field of light ( $\vec{e}$ is the polarization vector of the exciting light), $\left\langle v^{\prime} \mid v\right\rangle$ is the overlap matrix element for vibrational states of the ground and excited electronic states, $k_{B l t z}$ is the Boltzmann constant, $T$ is the absolute temperature, $\varepsilon_{10}=\varepsilon_{1}-\varepsilon_{0}$ and $\omega_{v^{\prime} v}=\omega_{v^{\prime}}-\omega_{v}$ are the differences in the energy levels of the electronic and vibrational states at the photoexciting light frequency $\omega=\varepsilon_{10}+\omega_{v^{\prime} v}$.

Since the light intensity is defined as $I_{\text {exp }}=E^{2}$, Eq. A.1 can be re-written as

$k_{\text {forward }}(\omega)=\alpha(\omega) I_{\text {exp }}$

in which the proportionality coefficient (parameter $\alpha$ ) is

$$
\begin{aligned}
\alpha(\omega)= & \frac{2 \pi}{\hbar}\left|\left\langle P_{1}|\vec{d} \vec{e}| P_{0}\right\rangle\right|^{2} \sum_{v} \sum_{v^{\prime}}\left|\left\langle v^{\prime} \mid v\right\rangle\right|^{2} \\
& \times \frac{\exp \left(-\omega_{v} / k_{B l t z} T\right)}{\sum_{v} \exp \left(-\omega_{v} / k_{B l t z} T\right)} \delta\left(\varepsilon_{10}+\omega_{v^{\prime} v}-\omega\right) .
\end{aligned}
$$

If multiple scattering effects occur, the actual electric field strength increases by the factor that equals the gain in the photoexcitation rate of each molecule. The $\alpha$ parameter in this case increases, in average, by the same factor.

\section{References}

Abgaryan G, Christophorov LN, Goushcha AO, Holzwarth AR, Kharkyanen VN, Knox PP, Lukashev EA (1998) Effects of mutual influence of photoinduced electron transitions and slow structural rearrangements in bacterial photosynthetic reaction centers. J Biol Phys 24:1-17 
Agmon N, Hopfield JJ (1983) Transient kinetics of chemical reactions with bounded diffusion perpendicular to the reaction coordinate: intermolecular processes with slow conformational changes. J Chem Phys 78:6947-6959

Cavatorta P, Casali E, Sartor G (1986) The problem of light scattering in the absorbance and fluorescence studies of proteins in membranes. In: Azzi A, Masotti L, Vecli A (eds) Membrane proteins: isolation and characterization. Springer, Berlin, pp 24-31

Clayton RK (1965) Molecular physics in photosynthesis. Blaisdell Pub Co, New York

Crofts AR (2004) The cytochrome $b_{1}$ complex: function in the context of structure. Annu Rev Physiol 66:689-733

Feher G, Okamura MY (1978) Chemical composition and properties of reaction centers. In: Clayton RK, Sistrom WR (eds) The photosynthetic bacteria. Plenum Press, New York, pp 349-386

Goldsmith JO, Boxer SG (1996) Rapid isolation of bacterial photosynthetic reaction centers with an engineered poly-histidine tag. Biochim Biophys Acta 1276(3):171-175

Goushcha AO, Kharkyanen VN, Scott GW, Holzwarth AR (2000) Self-regulation phenomena in bacterial reaction centers. 1. General theory. Biophys J 79:1237-1252

Goushcha AO, Manzo AJ, Scott GW, Christophorov LN, Knox PP, Barabash YM, Kapoustina MT, Berezetska NM, Kharkyanen VN (2003) Self-regulation phenomena applied to bacterial reaction centers 2 . Nonequilibrium adiabatic potential: dark and light conformations revisited. Biophys J 84:1146-1160

Goushcha AO, Manzo AJ, Kharkyanen VN, van Grondelle R, Scott GW (2004) Light-induced equilibration kinetics in membranebound photosynthetic reaction centers: nonlinear dynamic effects in multiple scattering media. J Phys Chem B 108(8):2717-2725

Hoff AJ, Deisenhofer J (1997) Photophysics of photosynthesis: structure and spectroscopy of reaction centers of purple bacteria. Phys Rep 287:1-247

Hudson RD (1969) Infrared system engineering. Wiley, New York

Jones MR, Visschers RW, van Grondelle R, Hunter CN (1992) Construction and characterization of a mutant of Rhodobacter sphaeroides with the reaction center as the sole pigment-protein complex. Biochemistry 31:4458-4465

Kleinfeld D, Okamura MY, Feher G (1984a) Electron transfer kinetics in photosynthetic reaction centers cooled to cryogenic temperatures in the charge-separated state: evidence of lightinduced structural changes. Biochemistry 23:5780-5786

Kleinfeld D, Okamura MY, Feher G (1984b) Electron transfer in reaction centers of Rhodopseudomonas sphaeroides. 1. Determination of the charge recombination pathway of $\mathrm{D}^{+} \mathrm{Q}_{\mathrm{A}}^{-} \mathrm{Q}_{\mathrm{B}}^{-}$and free-energy and kinetic relations between $\mathrm{Q}_{\mathrm{A}}^{-} \mathrm{Q}_{\mathrm{B}}$ and $\mathrm{Q}_{\mathrm{A}} \mathrm{Q}_{\mathrm{B}}^{-}$. Biochim Biophys Acta 766:126-140

Labahn A, Paddock ML, McPherson PH, Okamura MY, Feher G (1994) Direct charge recombination from $D^{+} Q_{A} Q_{B}^{-}$to $D_{A} Q_{B}$ in bacterial reaction centers from Rhodobacter sphaeroides. J Phys Chem 98:3417-3423

Lin S, Katilius E, Haffa ALM, Taguchi AKW, Woodbury NW (2001) Blue light drives B-side electron transfer in bacterial photosynthetic reaction centers. Biochemistry 40(46):13767-13773

Olenchuk MV, Barabash YM, Christophorov LN, Kharkyanen VN (2007) Peculiarities of light propagation through the media of molecules with long-lived photoexcited states. Chem Phys Lett 447:358-363

Shinkarev VP, Wraight CA (1997) The interaction of quinone and detergent with reaction centers of purple bacteria. 1. Slow quinone exchange between reaction center micelles and pure detergent micelles. Biophys J 72:2304-2319

Straley SC, Parson WW, Mauzerall DC, Clayton RK (1973) Pigment content and molar extinction coefficients of photochemical reaction centers from Rhodopseudomonas sphaeroides. Biochim Biophys Acta 305(5):597-609

Wraight CA (2004) Proton and electron transfer in the acceptor quinone complex of photosynthetic reaction centers from Rhodobacter sphaeroides. Front Biosci 9:309-337 